\title{
Vulnérabilité des familles dirigées par une mère adolescente ou jeune adulte faisant l'objet d'une enquête menée par un service de protection de l'enfance au Canada
}

\author{
W. Hovdestad, Ph. D.; M. Shields; G. Williams, M. Sc.; L. Tonmyr, Ph. D.
}

Cet article a fait l'objet d'une évaluation par les pairs.

Diffuser cet article sur Twitter

\section{Résumé}

Introduction : Les familles avec une jeune mère sont associées à un risque accru de mauvais traitements envers les enfants et de problèmes sociaux et de santé.

Méthodologie : Une analyse du chi carré effectuée sur des données combinées des services de protection de l'enfance issues de l'Étude canadienne sur l'incidence des signalements de cas de violence et de négligence envers les enfants (ECI-2003 et ECI2008) a permis de comparer 284 mères adolescentes (18 ans et moins) et 800 jeunes mères (19 à 21 ans) et leurs familles à 5752 familles avec une mère de 22 ans ou plus.

Résultats : Vingt-six pour cent des jeunes mères avaient 18 ans ou moins. La plupart recevaient de l'aide sociale comme principale source de revenu (68 \% des familles avec une mère adolescente et $57 \%$ des familles avec une mère jeune adulte contre $36 \%$ des familles avec une mère de 22 ans ou plus). Les mères adolescentes et les mères jeunes adultes étaient plus susceptibles d'avoir été placées dans leur enfance que les mères de 22 ans ou plus (31 \% et $23 \%$ contre $10 \%$ ) et présentaient plus fréquemment des facteurs de risque tels que l'alcoolisme ( $25 \%$ et $23 \%$ contre $18 \%$ ) ou un manque de soutien social (46\% et $41 \%$ contre $37 \%$ ). Les pourvoyeurs secondaires de soins dans les familles avec de jeunes mères étaient aussi associés à un plus grand nombre de facteurs de risque. Les familles de mères adolescentes ou jeunes adultes couraient un risque plus élevé de décision de placement de l'enfant pendant l'enquête ( $29 \%$ et $27 \%$ contre $17 \%$ ). Les mères couraient toutes le même risque d'être victimes de violence familiale et de présenter des problèmes de santé mentale.

Conclusion : Dans cet échantillon de familles à risque élevé, les risques étaient plus importants pour les familles avec de jeunes mères que pour les familles auxquelles on les avait comparées. Le jeune âge de la mère pourrait être un bon critère pour repérer les familles nécessitant des interventions ciblées.

Mots-clés : mauvais traitements envers les enfants, violence envers les enfants, violence familiale, mère adolescente, placement hors du foyer, Étude canadienne sur l'incidence des signalements de cas de violence et de négligence envers les enfants

\section{Introduction}

Les enfants nés de jeunes mères, qu'ils soient premier enfant ou suivant, et les mères elles-mêmes présentent un risque élevé de problèmes ${ }^{1,2}$. La maternité précoce constitue donc un sujet de préoccupation en santé, en éducation et en service social. Dans cet article, nous avons utilisé des données provenant d'un échantillon d'enquêtes sur les mauvais traitements envers les enfants menées au Canada en 2003 et en 2008 pour obtenir les caractéristiques des familles avec une

\section{Principales constatations}

- Les données des organismes de protection de l'enfance au Canada indiquent que les mères adolescentes connaissent de plus grandes difficultés que les mères plus âgées.

- Les mères adolescentes et les pourvoyeurs secondaires de soins à l'enfant qui vivent sous le même toit sont davantage susceptibles de :

- bénéficier de l'aide sociale,

- avoir été placés dans une famille d'accueil ou un foyer de groupe durant l'enfance,

- souffrir d'alcoolisme ou de toxicomanie,

- souffrir de troubles cognitifs,

- manquer de soutien social.

- Les mères adolescentes sont confrontées aux mêmes problèmes de santé mentale et d'exposition à la violence conjugale que les mères plus âgées.

- Dans le cadre de programmes visant la prévention des mauvais traitements envers les enfants, adopter une attitude proactive pour rejoindre les jeunes mères à risque qui subissent du stress sur de nombreux fronts serait sans doute nécessaire.

mère adolescente (18 ans ou moins) ou jeune adulte (19 à 21 ans) ayant fait l'objet d'un signalement aux services de protection de l'enfance et nous les avons comparées aux familles avec une mère de 22 ans ou plus. On s'intéresse aux facteurs de risque modifiables de ces familles (p. ex. alcoolisme, manque de soutien social) qui

Rattachement des auteurs :

Division de la surveillance et de l'épidémiologie, Agence de la santé publique du Canada, Ottawa (Ontario), Canada

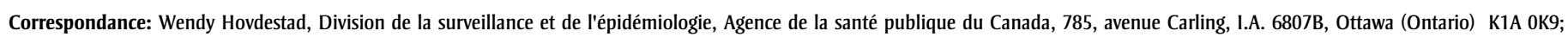
tél. : 613-799-0921; courriel : wendy.hovdestad@phac-aspc.gc.ca 
pourraient être ciblés au moyen d'interventions ou de programmes de soutien.

En 2010, le taux de naissances vivantes était de 7,7 pour 1000 femmes chez les mères de 15 à 17 ans et de 25,8 pour 1000 femmes chez les mères de 18 à 19 ans $^{3}$. Des différences notables ont été relevées quant au lieu de résidence de ces femmes : en 2006-2010, le taux de naissances vivantes par âge chez les femmes de 10 à 17 ans variait entre 1,6 pour 1000 au Québec et 29,4 pour 1000 au Nunavut ${ }^{3}$. Chez les femmes de 18 et 19 ans, ce taux variait entre 17,8 pour 1000 en Colombie-Britannique et 168,9 pour 1000 au Nunavut ${ }^{3}$. Les naissances associées à une mère de 20 ans ou plus représentaient $96 \%$ des naissances vivantes au Canada en $2009^{4}$. Selon Jutte et collab., 4,2 \% des naissances vivantes au Canada en 2006 étaient associées à des adolescentes ${ }^{2}$.

La variation du taux de maternité précoce entre les provinces et les territoires pourrait s'expliquer en partie par des différences culturelles liées à sa désirabilité. Les données probantes associées à des retombées positives pour les jeunes mères et leurs enfants et à l'importance des facteurs culturels influençant ces résultats ont été présentées dans d'autres publications ${ }^{5}$. Il n'en demeure pas moins que les enfants de jeunes mères courent au Canada un risque accru de mauvaise santé et de problèmes de développement ${ }^{2}$. Jutte et collab. ont étudié une cohorte de 32000 enfants nés entre 1979 et 1984 et résidants à Winnipeg à l'âge de 17 ans $^{2}$. Les enfants nés de mères adolescentes $(6 \%)$ et les enfants nés de mères adolescentes au moment de la naissance de leur premier enfant (10\%) ont été comparés aux enfants de femmes qui n'ont pas été mères à l'adolescence. Les enfants nés de mères adolescentes ou de mères ayant donné naissance à une sœur ou à un frère plus âgé pendant leur adolescence sont associés à un taux de mortalité de 2 à 4 fois plus élevé que les enfants des autres mères. On avait accès pour une partie de l'échantillon aux données sur les interventions des services de protection de l'enfance au moment où les enfants avaient entre 8 et 17 ans. Les enfants de mères adolescentes ou de mères ayant été mères à l'adolescence couraient 2 à 3 fois plus de risque d'avoir fait l'objet de ces interventions. La moitié de la cohorte d'enfants placés en famille d'accueil était constituée d'enfants de mères adolescentes ou de mères ayant été mères à l'adolescence.

L'analyse de données d'autres travaux de recherche canadiens effectués sur 1928 jeunes adultes en Ontario indique que ceux dont la mère était jeune (20 ans ou moins) lors de la naissance du premier enfant étaient davantage susceptibles d'avoir subi de la violence (physique ou sexuelle) durant leur enfance ${ }^{6}$. Cette association s'est maintenue même en contrôlant statistiquement le statut socioéconomique de la famille.

Les mauvais traitements infligés dans les familles dirigées par une jeune mère sont particulièrement préoccupants, car les nourrissons et les jeunes enfants maltraités sont très vulnérables aux blessures, risquent de mourir et sont souvent considérés comme suffisamment à risque pour requérir un placement hors de leur foyer ${ }^{7-9}$. Les enfants canadiens de 17 ans et moins décédés en raison d'un homicide commis par un membre de la famille sont d'ailleurs surtout des enfants de moins de 12 mois $^{9}$, ce qui est un indicateur de risque. Compte tenu de cette vulnérabilité, la nécessité d'une intervention immédiate des services sociaux pourrait être plus grande dans ces familles que dans celles où la mère et les enfants sont plus âgés.

Fallon et collab. ${ }^{10}$ ont utilisé les données du cycle de 2008 de l'Étude canadienne sur l'incidence des signalements de cas de violence et de négligence envers les enfants (ECI) pour répertorier les caractéristiques des jeunes parents canadiens dont la famille a fait l'objet d'une enquête par un service de protection de l'enfance et les caractéristiques des services prodigués à ces familles. D’après leurs résultats, $22 \%$ à $23 \%$ des mères biologiques de 21 ans ou moins présentaient des antécédents de placement en famille d'accueil ou en foyer de groupe, contre $10 \%$ des mères biologiques ayant entre 22 ans et 30 ans. Dans d'autres analyses réalisées sur les parents biologiques de moins de 31 ans s'occupant d'un enfant, les chercheurs ont observé que les antécédents de séjour en institution étaient associés à des services permanents à la famille, à un manque de soutien social et à des problèmes de santé mentale, d'alcoolisme ou de toxicomanie ${ }^{10}$.

De fait, les données populationnelles sur la protection de l'enfance indiquent que les familles avec de jeunes mères sont associées à un plus grand nombre de facteurs de risque liés aux mauvais traitements envers les enfants et à un plus grand besoin d'intervention de la part des services de protection de l'enfance. Nous avons combiné les données de l'ECI de 2003 (ECI-2003) et de l'ECI de 2008 (ECI-2008) pour dresser le portrait des familles canadiennes ayant fait l'objet d'un signalement aux services de protection de l'enfance. Les mères adolescentes et les jeunes mères et leur famille ont été comparées aux familles avec des mères de 22 ans ou plus.

\section{Méthodologie}

\section{Source des données}

Notre étude repose sur l'analyse de données recueillies au cours de deux cycles de l'ECI, celui de 2003 et celui de 2008. Les données des deux cycles ont été regroupées pour augmenter la taille de l'échantillon. L'ECI a répertorié les enquêtes sur les mauvais traitements envers des enfants menées par des organismes de protection de l'enfance du Canada sur trois mois, à l'automne de l'année d'étude. Les travailleurs des services de protection de l'enfance ont rempli un questionnaire de trois pages sur chaque enfant faisant l'objet d'une enquête, quatre à huit semaines après qu'une allégation ait été portée. L'ECI ne tient compte ni des dossiers déjà ouverts, ni des cas rejetés, ni des dossiers ouverts en raison de motifs autres que les mauvais traitements envers un enfant. Les données du Québec ont été exclues de l'ECI-2003 du fait de différences dans la méthodologie de collecte et dans la comparaison entre les données. De plus amples renseignements sur l'ECI-2003 et l'ECI-2008 sont disponibles dans d'autres publications ${ }^{11,12}$. L'appartenance de l'enfant et de son principal pourvoyeur de soins à un groupe autochtone est consignée dans l'ECI, mais la consultation auprès des peuples autochtones en vertu des principes PCAP (propriété, contrôle, accès et possession) n'ayant pas eu lieu, ces variables ont été exclues de l'analyse.

\section{Échantillon d'étude}

Nous avons sélectionné à partir des ensembles de données regroupées de l'ECI2003 et de l'ECI-2008 tous les enfants dont 
le principal pourvoyeur de soins était la mère biologique. Nous avons divisé l'échantillon en trois groupes en fonction de l'âge de la mère : mères adolescentes, de 18 ans ou moins $(n=284)$, mères jeunes adultes, de 19 à 21 ans $(n=800)$, et mères de 22 ans ou plus ( $n=5752$ ). Compte tenu des facteurs de stress liés au fait d'avoir un jeune enfant, et afin d'augmenter la comparabilité des groupes, nous n'avons retenu que les familles comptant au moins un enfant de moins de 6 ans. Lorsqu'il y avait plus d'un enfant de moins de 6 ans, nous avons axé l'étude sur le plus jeune enfant biologique.

\section{Mesures}

Le questionnaire comprenait des données sur l'enfant (âge et sexe), le ménage (sources de revenu, logement surpeuplé ou non), le principal pourvoyeur de soins et le pourvoyeur secondaire de soins de l'enfant (sexe, lien de parenté avec l'enfant et facteurs de risque), l'orientation vers un service par un travailleur des services de protection de l'enfance en raison d'un membre de la famille et enfin résultat de l'enquête. Les groupes d'âge des pourvoyeurs de soins de l'enfant étaient les suivants : moins de 16 ans, 16 à 18 ans, 19 à 21 ans, 22 à 30 ans, 31 à 40 ans, 41 à 50 ans, 51 à 60 ans, plus de 60 ans, ce qui a limité notre capacité à obtenir des données en fonction d'une ventilation plus détaillée de l'âge de la mère.

Le travailleur des services de protection de l'enfance a coté chacun des facteurs de risque comme étant «confirmé», « soupçonné », «non » ou « inconnu », à la fois pour le pourvoyeur principal et pour le pourvoyeur secondaire de soins à l'enfant. Nous avons combiné les pourcentages des facteurs «confirmés " et « soupçonnés » pour l'alcoolisme, la toxicomanie et l'inhalation de solvants, les troubles cognitifs, les problèmes de santé mentale, le manque de soutien social, le fait d'être victime de violence familiale, les antécédents de placement dans une famille d'accueil ou un foyer de groupe et le fait d'être auteur de violence familiale. Les cas pour lesquels le travailleur a coté « inconnu » ont été inclus dans le dénominateur (c.-à-d. qu'on a présumé que le pourvoyeur de soins de l'enfant ne présentait pas ce facteur de risque).

L'ECI-2008 a recensé deux types d'enquête : les enquêtes sur les mauvais traitements, pour lesquelles la personne ayant avisé les services de protection de l'enfance estimait qu'il y avait eu maltraitance, et les enquêtes sur le risque de futurs mauvais traitements, pour lesquelles la personne ayant avisé les services de protection de l'enfance estimait que l'enfant risquait de futurs mauvais traitements. L’ECI-2003 n'a intégré que les enquêtes sur les mauvais traitements. Lors de chaque enquête sur les mauvais traitements, le travailleur des services de protection de l'enfance est susceptible d'enquêter sur chacune des cinq grandes catégories de mauvais traitements (violence physique, abus sexuel, négligence, violence psychologique et exposition à la violence conjugale) et de la coter comme étant une préoccupation primaire, secondaire ou tertiaire. Le travailleur cote également chaque catégorie de mauvais traitements examinée comme étant «corroborée », « soupçonnée» ou «non corroborée». Nous avons présenté le pourcentage d'enquêtes (combinant les préoccupations primaires, secondaires et tertiaires) par catégorie de mauvais traitements et le pourcentage de mauvais traitements corroborés parmi ces enquêtes. L'enquête sur le risque de futurs mauvais traitements était considérée comme corroborée si le travailleur avait fait état d'un risque important de futurs mauvais traitements. Nous avons présumé que la réponse « inconnu » était un « non ».

\section{Analyses statistiques}

Nous avons utilisé des tests du chi carré pour déceler les variations entre les estimations relatives aux familles avec une jeune mère (adolescente ou jeune adulte) et celles relatives aux familles dont la mère avait 22 ans ou plus, en présumant que l'échantillon combiné de l'ECI avait été constitué de façon aléatoire. Comme nos travaux étaient axés sur les facteurs associés aux familles avec une jeune mère (plutôt que sur le nombre de familles avec de jeunes mères) et compte tenu des préoccupations soulevées par la représentativité au Canada des estimations fondées sur les données pondérées de $\mathrm{l}^{\prime} \mathrm{ECI}^{13}$, toutes les analyses ont été réalisées avec les données non pondérées. Ces analyses ont été menées en utilisant le SAS Enterprise Guide 5.1 (SAS Institute Inc., Cary, Caroline du Nord, É.-U.).

\section{Résultats}

Dans les familles où le principal pourvoyeur de soins de l'enfant était une jeune mère (moins de 21 ans), $26 \%$ de ces mères avaient 18 ans ou moins. La source la plus fréquente de revenu des ménages dont le principal pourvoyeur de soins de l'enfant était une jeune mère entrait dans la catégorie « aide sociale, autres types de prestations ou absence de revenu » (pour $68 \%$ des mères adolescentes de 18 ans ou moins et $57 \%$ des mères jeunes adultes de 19 à 21 ans contre $36 \%$ des familles dont la mère avait 22 ans ou plus) (tableau 1). Les familles avec une mère adolescente et les familles avec une mère de 22 ans ou plus bénéficiaient plus souvent de la présence à la maison d'un pourvoyeur secondaire de soins de l'enfant (respectivement $57 \%$ et $59 \%$ ) que les familles avec une mère jeune adulte (52\%). Un autre adulte (autre que les pourvoyeurs de soins principal et secondaire de l'enfant) vivait dans la maison de $51 \%$ des familles avec une mère adolescente, contre $29 \%$ pour les familles avec une mère jeune adulte et $15 \%$ pour les familles avec une mère de 22 ans ou plus. Dans $72 \%$ des familles avec une mère adolescente et un autre adulte sous le même toit, l'autre adulte était un grand-parent (données non présentées). Les familles avec une mère adolescente vivaient plus souvent dans un logement surpeuplé, et à la fois les familles avec une mère adolescente et les familles avec une mère jeune adulte avaient plus souvent déclaré plusieurs déménagements au cours de l'année.

Les jeunes mères étaient plus nombreuses que les mères de 22 ans ou plus à présenter des facteurs de risque recensés par le travailleur des services de protection de l'enfance responsable de l'enquête, notamment l'alcoolisme ou la toxicomanie, les troubles cognitifs et le manque de soutien social (tableau 2). Les mères adolescentes étaient plus nombreuses que les mères jeunes adultes à être associées à ces facteurs de risque. Les mères des trois groupes couraient le même risque d'être 
TABLEAU 1

Caractéristiques du ménage, de la famille et de l'enfant des familles recensées ${ }^{\mathrm{a}}$ dans lesquelles le principal pourvoyeur de soins de l'enfant est la mère biologique, 2003 et 2008

\begin{tabular}{|c|c|c|c|c|c|c|}
\hline \multirow[t]{3}{*}{ Caractéristiques } & \multicolumn{6}{|c|}{ Âge de la mère (ans) } \\
\hline & \multicolumn{2}{|c|}{18 ou moins } & \multicolumn{2}{|c|}{19 à 21} & \multicolumn{2}{|c|}{22 ou plus } \\
\hline & $\mathbf{N}$ & $\%$ & $\mathbf{N}$ & $\%$ & $\mathbf{N}$ & $\%$ \\
\hline Nombre total de familles & 284 & & 800 & & 5752 & \\
\hline \multicolumn{7}{|l|}{ Âge de la mère (ans) } \\
\hline Moins de 16 & 15 & 5,3 & - & - & - & - \\
\hline 16 à 18 & 269 & 94,7 & - & - & - & - \\
\hline 19 à 21 & - & - & 800 & 100,0 & - & - \\
\hline 22 à 30 & - & - & - & - & 3170 & 55,1 \\
\hline 31 à 40 & - & - & - & - & 2269 & 39,4 \\
\hline 41 et plus & - & - & - & - & 313 & 5,4 \\
\hline \multicolumn{7}{|l|}{ Âge de l'enfant (ans) } \\
\hline Nourrisson & 209 & $73,6^{*}$ & 382 & $47,8^{*}$ & 1265 & 22,0 \\
\hline 1 & 55 & 19,4 & 222 & $27,8^{*}$ & 1021 & 17,8 \\
\hline 2 & 15 & $5,3^{*}$ & 113 & 14,1 & 943 & 16,4 \\
\hline 3 & 5 & $1,8^{*}$ & 49 & $6,1^{*}$ & 836 & 14,5 \\
\hline 4 à 5 & $\mathrm{x}$ & $\mathrm{x}$ & 34 & $4,3^{*}$ & 1687 & 29,3 \\
\hline \multicolumn{7}{|l|}{ Sexe de l'enfant } \\
\hline Fille & 133 & 46,8 & 376 & 47,0 & 2848 & 49,5 \\
\hline \multicolumn{7}{|l|}{ Principale source de revenu du ménage $e^{b}$} \\
\hline Aide sociale/autre type de prestation/aucun revenu & 187 & $67,8^{*}$ & 448 & $57,4^{*}$ & 1984 & 36,0 \\
\hline Emplois multiples/emploi saisonnier/assurance-emploi & 8 & $2,9^{*}$ & 65 & $8,3^{*}$ & 345 & 6,3 \\
\hline Travail à temps partiel & 24 & 8,7 & 57 & 7,3 & 388 & 7,0 \\
\hline Travail à temps plein & 57 & $20,7^{*}$ & 211 & $27,0^{*}$ & 2796 & 50,7 \\
\hline $\begin{array}{l}\text { Pourvoyeur secondaire de soins de l'enfant vivant } \\
\text { sous le même toit }\end{array}$ & 162 & 57,0 & 418 & $52,3^{*}$ & 3366 & 58,5 \\
\hline Autre(s) adulte(s) vivant sous le même toit ${ }^{c}$ & 144 & $50,7^{*}$ & 230 & $28,8^{*}$ & 872 & 15,2 \\
\hline Logement surpeuplé & 38 & $13,4^{*}$ & 56 & 7,0 & 361 & 6,3 \\
\hline \multicolumn{7}{|l|}{ Nombre de déménagements au cours de l'année ${ }^{d}$} \\
\hline 0 & 72 & $32,6^{*}$ & 189 & $32,1^{*}$ & 2468 & 57,2 \\
\hline 1 & 75 & 33,9 & 231 & $39,3^{*}$ & 1212 & 28,1 \\
\hline 2 & 30 & $13,6^{*}$ & 82 & $13,9^{*}$ & 372 & 8,6 \\
\hline 3 ou plus & 44 & $19,9^{*}$ & 86 & $14,6^{*}$ & 260 & 6,0 \\
\hline
\end{tabular}

Source : Étude canadienne sur l'incidence des signalements de cas de violence et de négligence envers les enfants, 2003 et 2008. Remarque : Estimation reposant sur le plus jeune enfant biologique si plus d'un enfant de la famille fait l'objet d'une enquête.

a L'étude ne portait que sur les familles comptant au moins un enfant âgé de moins de 6 ans. Lorsqu'il y avait plus d'un enfant de moins de 6 ans, l'enfant biologique le plus jeune a été choisi.

b Données manquantes sur la principale source de revenu du ménage exclues (pour 8 mères de 18 ans ou moins, 19 mères de 19 à 21 ans et 239 mères de 22 ans ou plus).

c À l'exception des premiers et deuxièmes pourvoyeurs de soins de l'enfant.

d Données manquantes sur le nombre de déménagements exclues (pour 63 mères de18 ans ou moins, 212 mères de 19 à 21 ans et 1440 mères de 22 ans ou plus).

* Significativement différent des mères de 22 ans ou plus $(p<0,05)$.

x Nombre de cellules inférieur à 5 .

évaluées comme présentant des problèmes de santé mentale et comme étant victimes de violence familiale. Les mères de 22 ans ou plus étaient plus souvent évaluées comme ayant des problèmes de santé physique. Les jeunes mères avaient plus souvent des antécédents de placement en famille d'accueil ou dans un foyer de groupe $(31 \%$ des mères adolescentes et $23 \%$ des mères jeunes adultes) que les mères de 22 ans ou plus (10\%). Les facteurs de risque multiples étaient plus fréquents chez les jeunes mères: trois facteurs de risque ou plus ont été décelés chez $30 \%$ des mères adolescentes et chez $29 \%$ des mères jeunes adultes, contre chez $23 \%$ des mères de 22 ans ou plus.

Dans les familles où un pourvoyeur secondaire de soins de l'enfant vivait sous le même toit (tableau 3), cette personne était le plus souvent le père biologique (pour $61 \%$ des mères adolescentes, $69 \%$ des mères jeunes adultes et $77 \%$ des mères de 22 ans ou plus), suivi de la grand-mère dans les familles avec une mère adolescente (25\%) et du beau-père ou du conjoint de la mère dans les familles avec une mère jeune adulte $(13 \%)$ et dans les familles avec une mère de 22 ans ou plus (17\%). Dans les familles avec une jeune mère, ce pourvoyeur secondaire de soins de l'enfant présentait souvent des facteurs de risque recensés par le travailleur social, notamment l'alcoolisme ou la toxicomanie, des troubles cognitifs, un manque de soutien social ou des antécédents de placement dans une famille d'accueil ou un foyer de groupe. Dans tous les cas, ces facteurs de risque avaient été décelés plus fréquemment dans les familles avec une mère adolescente que dans les familles avec une mère jeune adulte. Le risque de présenter un problème de santé mentale ou physique était en revanche le même dans tous les types de familles. La présence de trois facteurs de risque ou plus chez ce pourvoyeur secondaire de soins avait été décelée dans plus du quart $(27 \%)$ des familles avec une mère adolescente, contre $23 \%$ dans les familles avec une mère jeune adulte et $14 \%$ dans les familles avec une mère de 22 ans ou plus.

Les enfants des familles avec une mère adolescente étaient plus souvent susceptibles de faire l'objet d'une enquête sur le risque de futurs mauvais traitements que ceux des familles dont la mère avait 22 ans ou plus (31\% contre $17 \%$; tableau 4 ). Les enquêtes sur le risque de futurs mauvais traitements étaient corroborées dans la même mesure dans les trois types de familles. Quoique ces enfants aient moins souvent fait l'objet d'une enquête sur les mauvais traitements que ceux vivant dans une famille avec une mère de 22 ans ou plus, le risque que les mauvais traitements 
TABLEAU 2

Facteurs de risque de la mère dans les familles recensées ${ }^{a}$ dans lesquelles le principal pourvoyeur de soins de l'enfant est la mère biologique

\begin{tabular}{|c|c|c|c|c|c|c|}
\hline \multirow[t]{3}{*}{ Facteurs de risque } & \multicolumn{6}{|c|}{ Âge de la mère (ans) } \\
\hline & \multicolumn{2}{|c|}{18 ou moins } & \multicolumn{2}{|c|}{19 à 21} & \multicolumn{2}{|c|}{22 ou plus } \\
\hline & $\mathbf{N}$ & $\%$ & $\mathbf{N}$ & $\%$ & $\mathbf{N}$ & $\%$ \\
\hline Nombre total de familles & 284 & & 800 & & 5752 & \\
\hline Alcoolisme & 72 & $25,4^{*}$ & 184 & $23,0^{*}$ & 1051 & 18,3 \\
\hline Toxicomanie/inhalation de solvants & 94 & $33,1^{*}$ & 229 & $28,6^{*}$ & 1069 & 18,6 \\
\hline Troubles cognitifs & 48 & $16,9^{*}$ & 92 & $11,5^{*}$ & 414 & 7,2 \\
\hline Problèmes de santé mentale & 69 & 24,3 & 212 & 26,5 & 1403 & 24,4 \\
\hline Problèmes de santé physique & 10 & $3,5^{*}$ & 40 & $5,0^{*}$ & 427 & 7,4 \\
\hline Manque de soutien social & 131 & $46,1^{*}$ & 327 & $40,9^{*}$ & 2106 & 36,6 \\
\hline Victime de violence familiale & 103 & 36,3 & 359 & 44,9 & 2414 & 42,0 \\
\hline $\begin{array}{l}\text { Antécédents de placement dans une famille d'accueil } \\
\text { ou un foyer de groupe }{ }^{b}\end{array}$ & 55 & $30,9^{*}$ & 112 & $23,3^{*}$ & 347 & 10,0 \\
\hline \multicolumn{7}{|l|}{ Nombre total de facteurs de risque recensés ${ }^{c}$} \\
\hline 0 & 51 & $18,0^{*}$ & 164 & $20,5^{*}$ & 1661 & 28,9 \\
\hline 1 & 86 & 30,3 & 231 & 28,9 & 1561 & 27,1 \\
\hline 2 & 63 & 22,2 & 172 & 21,5 & 1207 & 21,0 \\
\hline 3 ou plus & 84 & $29,6^{*}$ & 233 & $29,1^{*}$ & 1323 & 23,0 \\
\hline
\end{tabular}

Source : Étude canadienne sur l'incidence des signalements de cas de violence et de négligence envers les enfants, 2003 et 2008.

a L'étude ne portait que sur les familles comptant au moins un enfant âgé de moins de 6 ans.

b Uniquement interrogé en 2008 (178 mères de 18 ans ou moins, 480 mères de 19 à 21 ans et 3479 mères âgées de 22 ans ou plus).

c Selon sept facteurs (alcoolisme, toxicomanie ou inhalation de solvants, troubles cognitifs, problèmes de santé mentale, manque de soutien social, victime de violence familiale, problèmes de santé physique).

* Significativement différent des mères de 22 ans ou plus $(p<0,05)$.

soient corroborés était le même. Les enfants de familles avec une mère adolescente ou une mère jeune adulte avaient plus souvent fait l'objet d'une enquête pour négligence, tandis que les enfants de familles avec une mère de 22 ans ou plus avaient plus souvent fait l'objet d'une enquête menée en raison de violence physique ou d'abus sexuel. Les mêmes tendances ont été observées lorsque l'analyse se limitait aux familles dans lesquelles l'enfant était âgé de deux ans ou moins (données non présentées).

Les enquêtes sur la violence psychologique envers les enfants étaient plus fréquentes dans les familles avec une mère jeune adulte que dans les familles avec une mère de 22 ans ou plus (18\% contre $15 \%$ ). L'exposition à la violence conjugale constituait moins souvent l'objet d'une enquête chez les enfants de mère adolescente (20\%) que chez les enfants dont la mère avait 22 ans ou plus (29\%), mais ce type d'enquête était plus fréquent chez les enfants de mère jeune adulte (33\%). La même tendance a été constatée lorsque l'analyse se limitait aux familles dans lesquelles le père biologique ou le conjoint de la mère habitait sous le même toit (données non présentées). Les mauvais traitements faisant l'objet de l'enquête étaient corroborés dans la même mesure pour chacun des cinq types de mauvais traitements chez les enfants des trois groupes de famille (tableau 4).

Dans les familles où les mauvais traitements envers l'enfant le plus jeune avaient été corroborés, le travailleur social orientait plus fréquemment les jeunes mères (adolescentes ou jeunes adultes) que les mères de 22 ans ou plus vers un groupe de soutien ou un counselling pour parents ou encore vers du soutien familial à domicile (tableau 5). Les familles avec une mère adolescente étaient plus souvent orientées vers de l'aide sociale, et les familles avec une mère jeune adulte vers des services médicaux ou dentaires. Les familles avec une mère adolescente étaient moins fréquemment orientées vers des services d'aide aux victimes de violence familiale. La même tendance (orientations en raison de violence familiale presque aussi nombreuses dans les familles avec une mère jeune adulte que dans les familles du groupe de comparaison et moins nombreuses dans les familles avec une mère adolescente) a été observée lorsque l'analyse se limitait aux familles dont le père biologique ou le conjoint de la mère vivait sous le même toit (données non présentées). Les membres de la famille étaient susceptibles dans la même mesure dans les trois groupes d'être dirigés vers un counselling en matière de toxicomanie ou d'alcoolisme, une banque alimentaire ou un refuge, ou encore vers des services psychiatriques ou psychologiques.

Parmi les familles avec une jeune mère dans lesquelles la mère ou le pourvoyeur secondaire de soins de l'enfant avait été évalué comme alcoolique ou toxicomane, moins d'un tiers ont été dirigées vers un counselling en matière de toxicomanie ou d'alcoolisme (26\% dans les familles avec une mère adolescente, $29 \%$ dans les familles avec une mère jeune adulte et $31 \%$ dans les familles avec une mère de 22 ans ou plus). Dans les familles avec une jeune mère où des problèmes de santé mentale chez la mère ou le pourvoyeur secondaire de soins de l'enfant avaient été relevés, moins d'un quart de ces derniers ont été orientés vers des services psychiatriques ou psychologiques (19\% dans les familles avec mère adolescente, $24 \%$ dans les familles avec mère jeune adulte, $23 \%$ dans les familles avec mère de 22 ans ou plus) (données non présentées).

Les enfants de mère adolescente étaient plus souvent soumis dans le cadre de l'enquête à un examen physique réalisé par un médecin ou une infirmière que les enfants du groupe de comparaison. Le placement de l'enfant durant l'enquête était plus fréquent pour les familles avec une jeune mère (adolescente ou jeune adulte) que pour les familles avec une mère de 22 ans ou plus (29\% et $27 \%$ contre $17 \%$ ). La police intervenait moins souvent dans le cadre d'une enquête pour violence familiale entre adultes dans les familles avec une mère adolescente, et cette tendance se maintenait lorsque l'analyse se limitait aux familles dans lesquelles le père biologique ou le conjoint de la mère vivait sous le même toit (données non présentées). Les trois groupes étaient susceptibles dans la 
TABLEAU 3

Caractéristiques du pourvoyeur secondaire de soins de l'enfant dans les familles recensées ${ }^{\mathrm{a}}$ dans lesquelles le principal pourvoyeur de soins de l'enfant est la mère biologique

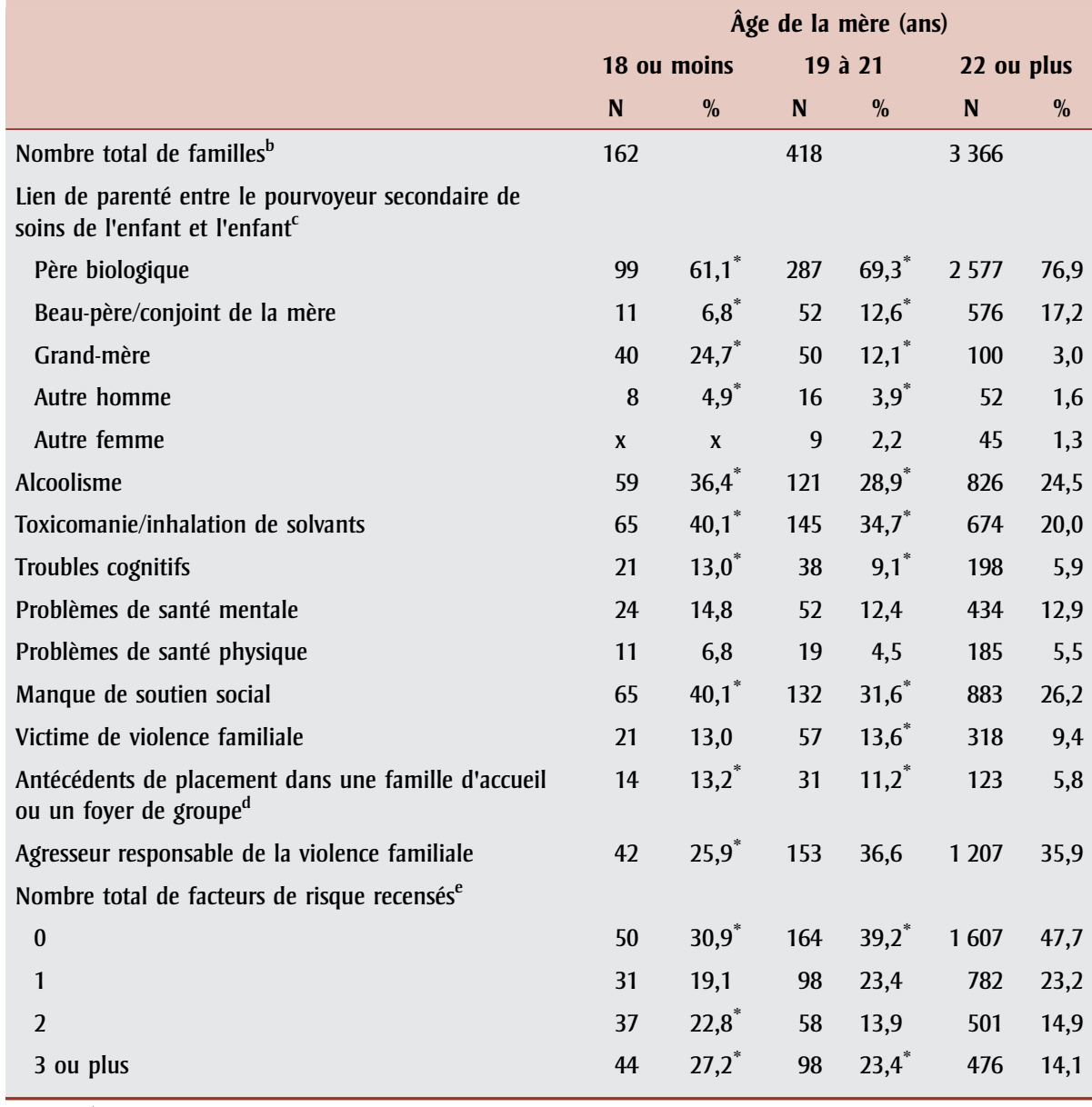

Source : Étude canadienne sur l'incidence des signalements de cas de violence et de négligence envers les enfants, 2003 et 2008.

Remarque : Les estimations reposent sur l'enfant biologique le plus jeune si plus d'un enfant de la famille fait l'objet d'une enquête.

a L'étude ne portait que sur les familles comptant au moins un enfant âgé de moins de 6 ans. Lorsqu'il y avait plus d'un enfant de moins de 6 ans, l'enfant biologique le plus jeune a été choisi.

b Familles dont le pourvoyeur secondaire de soins de l'enfant vit sous le même toit.

c Sauf les cas pour lesquels on ne connaît pas le lien entre le pourvoyeur secondaire de soins de l'enfant et l'enfant (pour 4 mères de 19 à 21 ans et 16 mères de 22 ans ou plus).

${ }^{\text {d }}$ Uniquement demandé en 2008 (pour 106 mères de 18 ans ou moins, 276 mères de 19 à 21 ans et 2133 mères de 22 ans ou plus).

e Selon sept facteurs (alcoolisme, toxicomanie ou inhalation de solvants, troubles cognitifs, problèmes de santé mentale, manque de soutien social, victime de violence familiale, problèmes de santé physique).

* Significativement différent des mères de 22 ans ou plus $(p<0,05)$.

$\mathrm{x}$ Nombre de cellules inférieur à 5 .

même mesure d'être la cible d'une intervention policière menée dans le cadre d'une enquête pour mauvais traitements envers un enfant.

\section{Analyse}

Les risques se sont révélés élevés que les membres de la famille faisant l'objet d'une enquête par les services de protection de l'enfance soient violents et présentent des problèmes de santé mentale, d'alcoolisme ou de toxicomanie et d'autres problèmes. Dans ce regroupement d'échantillons de familles canadiennes à risque élevé recensées en 2003 ou 2008, nous avons analysé, en fonction de l'âge de la mère, certaines variables liées à ces risques et portant sur le ménage, les caractéristiques de l'enfant et de la famille, les facteurs de risque de la mère et du pourvoyeur secondaire de soins de l'enfant et enfin les services ayant fait l'objet d'une orientation et utilisés. Dans tous les cas, on a observé un gradient d'âge évident, les familles dont les mères étaient les plus jeunes étant les plus à risque. Toutefois, aucun gradient d'âge de la mère n'était clairement visible quant aux types de mauvais traitements corroborés, et le lien entre l'âge de la mère et la violence conjugale était complexe.

Contrairement à d'autres échantillons dans lesquels les jeunes couples étaient plus portés que les couples plus âgés à faire état de violence ${ }^{14,15}$, les mères les plus jeunes de cet échantillon de données sur la protection de l'enfance n'étaient pas plus nombreuses à être victimes de violence familiale. À notre avis, ce résultat n'était pas attribuable à la présence ou à l'absence d'un conjoint, mais plutôt à la présence d'un autre adulte dans la maison, ce qui était très fréquent dans les familles avec une mère adolescente. Bien que le risque de violence conjugale des mères adolescentes puisse être élevé, la présence d'un autre adulte sous le même toit en réduirait les manifestations.

Le gradient d'âge de l'un des facteurs de risque des mères était particulièrement net : $10 \%$ des mères de 22 ans ou plus présentaient des antécédents de placement dans une famille d'accueil ou en foyer de groupe avérés ou soupçonnés, contre plus de $20 \%$ des mères de 19 à 21 ans et plus de $30 \%$ des mères de moins de 18 ans. Un gradient d'âge significatif mais plus faible a été relevé concernant le pourvoyeur secondaire de soins de l'enfant. Un séjour en famille d'accueil ou en foyer de groupe est l'indice d'antécédents de mauvais traitements dans l'enfance. Le lien entre des antécédents de mauvais traitements et une parentalité précoce a déjà été examiné $^{16-18}$. À l'aide des données tirées d'une étude longitudinale menée sur un échantillon de 1000 adolescents américains suivis depuis 1988 , Thornberry et collab. ${ }^{19}$ ont constaté que les mauvais traitements (y compris le fait d'être témoin d'actes graves de violence familiale) infligés au cours de la croissance étaient un facteur de risque lié au signalement corroboré de mauvais traitements envers un enfant avant l'âge 
TABLEAU 4

Type d'enquête(s) dont l'enfant le plus jeune ${ }^{a}$ fait l'objet dans les familles recensées dans lesquelles le principal pourvoyeur de soins de I'enfant est la mère biologique

\begin{tabular}{|c|c|c|c|c|c|c|c|c|c|c|c|c|}
\hline & \multicolumn{6}{|c|}{$\begin{array}{l}\text { Âge de la mère biologique (ans) ayant fait l'objet } \\
\text { d'une enquête }\end{array}$} & \multicolumn{6}{|c|}{$\begin{array}{l}\text { Âge de la mère biologique (ans) dont l'enquête a } \\
\text { corroboré le signalement }\end{array}$} \\
\hline & \multicolumn{2}{|c|}{18 ou moins } & \multicolumn{2}{|c|}{19 à 21} & \multicolumn{2}{|c|}{22 ou plus } & \multicolumn{2}{|c|}{18 ou moins } & \multicolumn{2}{|c|}{19 à 21} & \multicolumn{2}{|c|}{22 ou plus } \\
\hline & $\mathbf{N}$ & $\%$ & $\mathbf{N}$ & $\%$ & $\mathbf{N}$ & $\%$ & $\mathbf{N}$ & $\%^{d}$ & $\mathbf{N}$ & $\%^{d}$ & $\mathbf{N}$ & $\%^{d}$ \\
\hline Total & 284 & & 800 & & 5752 & & & & & & & \\
\hline Enquête sur le risque $^{\mathrm{b}}$ & 89 & $31,3^{*}$ & 146 & 18,3 & 974 & 16,9 & 31 & 34,8 & 46 & 31,5 & 260 & 26,7 \\
\hline Enquête sur les mauvais traitements & 195 & $68,7^{*}$ & 654 & 81,8 & 4778 & 83,1 & 111 & 56,9 & 360 & 55,0 & 2462 & 51,5 \\
\hline \multicolumn{13}{|c|}{ Type d'enquête sur les mauvais traitements ${ }^{c}$} \\
\hline Négligence & 129 & $45,4^{*}$ & 359 & $44,9^{*}$ & 2075 & 36,1 & 65 & 50,4 & 159 & 44,3 & 968 & 46,7 \\
\hline Exposition à la violence conjugale & 56 & $19,7^{*}$ & 262 & $32,8^{*}$ & 1680 & 29,2 & 36 & 64,3 & 189 & 72,1 & 1192 & 71,0 \\
\hline Violence psychologique & 38 & 13,4 & 142 & $17,8^{*}$ & 854 & 14,8 & 20 & 52,6 & 72 & 50,7 & 385 & 45,1 \\
\hline Violence physique & 25 & $8,8^{*}$ & 67 & $8,4^{*}$ & 1047 & 18,2 & 8 & 32,0 & 12 & 17,9 & 304 & 29,0 \\
\hline Abus sexuel & $x$ & $x$ & 11 & $1,4^{*}$ & 301 & 5,2 & $x$ & $x$ & $x$ & $x$ & 51 & 16,9 \\
\hline
\end{tabular}

Source : Étude canadienne sur l'incidence des signalements de cas de violence et de négligence envers les enfants, 2003 et 2008.

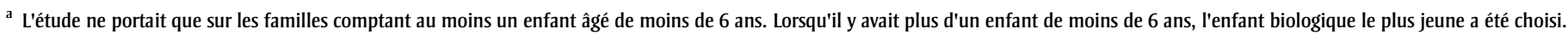

b Enquêtes sur le risque dont les données n'ont été recueillies qu'en 2008.

c Comprend les enquêtes menées en raison de mauvais traitements de forme primaire, secondaire et tertiaire.

${ }^{\mathrm{d}}$ Nombre de cas corroborés divisé par le nombre de cas ayant fait l'objet d'une enquête.

* Significativement différent des mères âgées de 22 ans ou plus $(p<0,05)$.

x Nombre de cellules inférieur à 5 .

de 33 ans. Dans cet échantillon, presque exclusivement masculin, une transition rapide vers l'âge adulte (c.-à-d. vivre en couple avant l'âge de 19 ans ou devenir parent avant l'âge de 20 ans) a été associée à un risque quatre fois plus élevé de perpétrer des mauvais traitements ${ }^{19}$.

Par conséquent, chez les jeunes mères et les pourvoyeurs secondaires de soins de l'enfant répertoriés dans cet échantillon, des antécédents personnels de mauvais traitements durant l'enfance pourraient être associés à la fois à la parentalité précoce et à l'implication des services de protection de l'enfance. Un programme d'intervention précoce auprès des jeunes mères du type de celles de cet échantillon pourrait être plus efficace, plus rentable et moins stigmatisant que les interventions des services de protection de l'enfance qui, par définition, ne surviennent que si une personne inquiète a signalé le cas aux services de protection de l'enfance.

Un gradient d'âge de la mère a été observé pour ce qui est de l'alcoolisme ou de la toxicomanie, les mères les plus jeunes et les pourvoyeurs secondaires de soins de l'enfant dans les familles avec de jeunes mères présentant plus fréquemment ces problèmes. Pourtant, le degré d'orientation vers un counselling en matière de toxicomanie ou d'alcoolisme était le même dans les trois groupes. Les mères et les pourvoyeurs secondaires de soins de l'enfant des jeunes familles auraient peut-être davantage besoin d'être orientés vers ces services. Il se peut aussi que ces personnes ne soient pas dirigées plus fréquemment vers ces services car elles en bénéficient déjà ou parce que ces services ne sont pas accessibles aux jeunes familles dans leur collectivité.

Nos travaux peuvent contribuer à l'étude des besoins potentiellement non comblés en matière de services et sur les meilleures façons de satisfaire ces besoins. Notre analyse peut en outre contribuer à éviter à la base une intervention des services de protection de l'enfance dans les familles. Ne faire l'objet d'aucune intervention des services de protection de l'enfance signifierait éviter les premiers mauvais traitements envers les enfants, et, idéalement, éviter également les facteurs de risque pouvant aboutir à une orientation vers les services de protection de l'enfance. Comme les mères les plus jeunes ont parfois des besoins multiples et subissent de nombreux stress qui font en sorte qu'il leur est difficile de bénéficier des services et du soutien qu'offrent la collectivité, les programmes visant la prévention des mauvais traitements pourraient devoir être proactifs pour rejoindre les mères à risque. En effet, les mères capables d'accéder aux services de soutien (p. ex. déposer leur nourrisson à une halte-garderie) pourraient ne pas être celles qui auraient le plus besoin de soutien.

Aux États-Unis, le Nurse-Family Partnership a ainsi réduit l'ampleur de plusieurs problèmes, notamment la mortalité de la mère et de l'enfant, comme deux décennies de suivi l'ont montré ${ }^{20}$. Ce partenariat est un programme intensif de visites à domicile au cours desquelles des infirmières spécialement formées en santé publique suivent de jeunes nouvelles mères, de la période prénatale jusqu'au 2 ans de l'enfant ${ }^{21}$. Toutes les mères participant au programme consentent à le faire et, dans ce cadre volontaire, une infirmière s'assure de la participation active de chacune. Les essais cliniques randomisés (ECR) menés aux États-Unis ont révélé que ce programme diminue le nombre de signalements de mauvais traitements envers les enfants vérifiés par l'État $^{22}$ ainsi que les facteurs de risque associés aux mauvais traitements envers les enfants ${ }^{21,23}$. Un ECR réalisé dans le cadre de ce programme est actuellement 
TABLEAU 5

Services financés par le secteur public utilisés par les familles recensées ${ }^{\mathrm{a}}$ ou vers lesquels on les a dirigés, familles dans lesquelles le principal pourvoyeur de soins de l'enfant est la mère biologique et pour lesquelles les mauvais traitements de l'enfant le plus jeune ont été corroborés

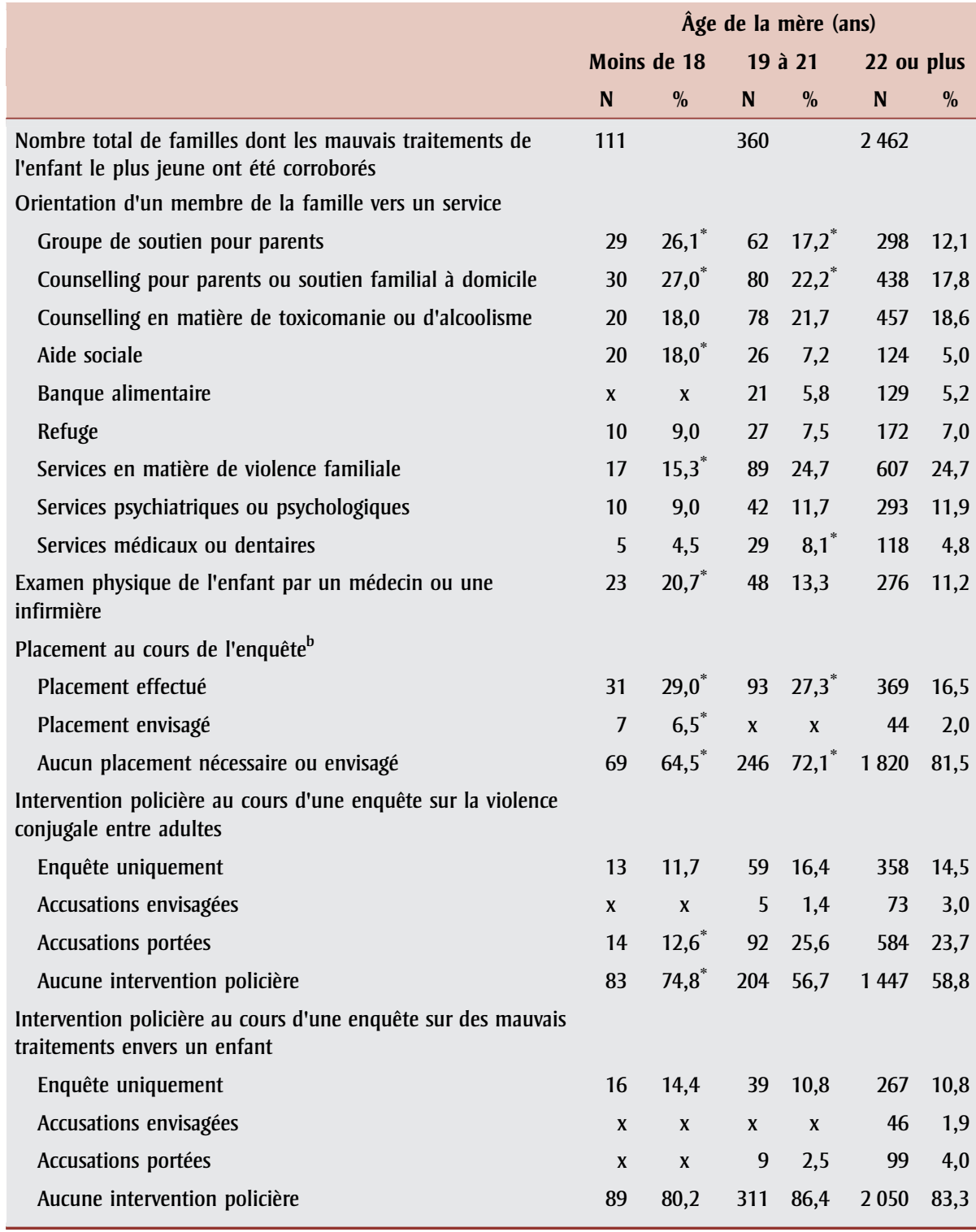

Source : Étude canadienne sur l'incidence des signalements de cas de violence et de négligence envers les enfants, 2003 et 2008.

${ }^{a}$ L'étude ne portait que sur les familles comptant au moins un enfant âgé de moins de 6 ans. Lorsqu'il y avait plus d'un enfant de moins de 6 ans, l'enfant biologique le plus jeune a été choisi.

b Sauf les cas de 2008 pour lesquels on a recueilli des données limitées sur le placement à certains sites (pour 23 mères de moins de 22 ans et 229 mères de 22 ans ou plus).

* Significativement différent des mères de 22 ans ou plus $(p<0,05)$.

x Nombre de cellules inférieur à 5 .

en cours en Colombie-Britannique pour déterminer si ce programme est efficace dans le contexte canadien ${ }^{24}$. Pour compléter l'ECR, l'Agence de la santé publique du Canada a commandé une évaluation de processus pour comprendre comment et pourquoi ce programme est une réussite ou un échec en fonction des différents contextes en Colombie-Britannique.

Comparativement à d'autres familles ayant fait l'objet d'un signalement auprès des services de protection de l'enfance, les familles avec une jeune mère présentent plus souvent des facteurs de risque modifiables par des programmes d'intervention. Ces jeunes mères ont des besoins qui, avec une intervention efficace plus précoce, pourraient ne pas avoir été pressants au point de nécessiter une intervention des services de protection de l'enfance. D'après Fallon et collab. $^{10}$, la prestation permanente d'un service à la suite d'une enquête visant la protection de l'enfant s'est révélée plus fréquente chez les jeunes mères, et ces prestations comportent des coûts. Il serait peut-être utile d'examiner le ratio coûtsavantages (autant pour les coûts humains que financiers) de la prestation précoce et ciblée de services de prévention aux familles les plus à risque par rapport à la prestation ultérieure d'interventions des services de protection de l'enfance. Les interventions précoces permettant de soutenir les jeunes mères aux prises avec des problèmes de toxicomanie et de santé mentale pourraient non seulement prévenir les futures enquêtes des services de protection de l'enfance mais aussi présenter des avantages à long terme pour la santé des mères. Cette information pourrait s'avérer utile à ceux qui cherchent à déterminer la rentabilité de la mise en œuvre d'un programme.

\section{Points forts et limites}

Comme les antécédents génésiques de la femme n'ont pas été pris en compte dans l'ECI, l'une des limites de nos analyses concerne le fait que certaines des mères de 22 ans ou plus pourraient avoir été mère au cours de leur adolescence. Nous n'avons pas été en mesure d'examiner les caractéristiques des familles dans lesquelles les mères avaient été mères à l'adolescence. Par ailleurs, la mesure des conditions socioéconomiques recensées dans cet ensemble de données était limitée, et nos analyses ne pouvaient pas prendre en compte les effets possibles du soutien social offert en situation de grossesse précoce selon la culture sur les facteurs de risque évalués dans le cadre de l'ECI. En outre, nous avons présumé qu'un échantillonnage aléatoire simple avait servi à la constitution de l'échantillon, ce qui implique probablement une sous-estimation de la variance lors du calcul des estimations.

L'une des forces de nos travaux de recherche est le fait que le groupe de comparaison avec 
les mères adolescentes et les mères jeunes adultes ayant déjà fait l'objet d'un signalement aux services de protection de l'enfance était constitué de mères ayant également fait l'objet d'un signalement auprès de ces services. En outre, nous avons constitué les groupes les plus semblables possible en restreignant les analyses aux familles comptant de jeunes enfants. Les jeunes mères ayant fait l'objet d'un signalement auprès des services de protection de l'enfance peuvent en effet être très différentes des jeunes mères répertoriées dans les enquêtes menées sur une population représentative de la population générale. Par exemple, dans le cas de la consommation d'alcool par les jeunes mères, les données de 2005 à 2008 indiquent que les mères de 20 à 24 ans (les plus jeunes de l'étude) étaient moins ou aussi nombreuses que les mères plus âgées à déclarer consommer de l'alcool au cours de la grossesse (8\% contre $11 \%$ en moyenne pour l'ensemble des groupes d'âge disponibles) ${ }^{3}$. À l'opposé, on a relevé un problème d'alcoolisme chez bon nombre des jeunes mères de l'échantillon utilisé. On est ainsi mieux à même de comprendre les besoins de ces jeunes familles en comparant ces mères à un groupe ayant fait l'objet d'un signalement aux services de protection de l'enfance plutôt qu'à la population générale.

\section{Références}

1. Koniak-Griffin D, Turner-Pluta C. Health risks and psychosocial outcomes of early childbearing: a review of the literature. J Perinat Neonat Nurs. 2001;15(2):1-17.

2. Jutte DP, Roos NP, Brownell MD, Briggs G, MacWilliam L, Roos LL. The ripples of adolescent motherhood: social, educational, and medical outcomes for children of teen and prior teen mothers. Acad Pediatr. 2010;10(5):293-301.

3. Agence de la santé publique du Canada. Indicateurs de la santé périnatale au Canada 2013. Ottawa (Ont.) : Agence de la santé publique du Canada; 2013.

4. Statistique Canada. Tableau 2-2 : naissances vivantes, selon la géographie - âge de la mère. Ottawa (Ont.) : Statistique Canada; 2009. [Statistique Canada, $\mathrm{N}^{\mathrm{O}} 84 \mathrm{~F} 0210 \mathrm{X}$ au catalogue]. Consultable en ligne à la page : http://www.statcan.gc.ca/pub/84f0210x/ 2009000/t003-fra.htm.
5. Sherraden MS, Gonzalez R, Rainford W. Hacia un futuro más seguro: pregnancy and childbearing among Latina adolescents. Dans Holgate HS, Evans R, Yuen FKO editors. Teenage pregnancy and parenthood: global perspectives, issues, and interventions. New York (NY) : Routledge; 2006 p. 36-57.

6. MacMillan HL, Tanaka M, Duku E, Vaillancourt T, Boyle MH. Child physical and sexual abuse in a community sample of young adults: results from the Ontario Child Health Study. Child Abuse Negl. 2013;37 (1):14-21

7. Tonmyr L, Williams G, Jack SM, MacMillan HL. Infant placement in Canadian child maltreatment-related investigations. Int $\mathrm{J}$ Ment Health Addict. 2011;9:441-459.

8. Williams G, Tonmyr L, Jack SM, Fallon B, MacMillan HL. Determinants of maltreatment substantiation in a sample of infants involved with the child welfare system. Child Youth Serv Rev. 2011;33:1345-1353.

9. Superle T. La violence familiale envers les enfants et les jeunes. Dans: La violence familiale au Canada : un profil statistique, 2013. Ottawa (Ont.) : Centre canadien de la statistique juridique; 2015. p. 46-62. [Statistique Canada, $\mathrm{N}^{\mathrm{o}}$ 85-002-X au catalogue].

10. Fallon B, Ma J, Black T, Wekerle C. Characteristics of young parents investigated and opened for ongoing services in child welfare. Int J Ment Health Addict. 2011;9(4):365-381.

11. Agence de la santé publique du Canada. Étude canadienne sur l'incidence des signalements de cas de violence et de négligence envers les enfants - 2008 : données principales. 2010. Ottawa (Ont.) : Agence de la santé publique du Canada; 2010.

12. Trocmé N, Fallon B, MacLaurin B et collab. Étude canadienne sur l'incidence des signalements de cas de violence et de négligence envers les enfants - 2003 : données principales. Ottawa (Ont.) : Agence de santé publique du Canada; 2005.

13. Dumais J. Comparison and assessment of CIS 1998, 2003 and 2008 Designs. 2010. Ottawa (Ont.) : Statistique Canada. Rapport non publié.

14. Al-Sahab B, Heifetz M, Tamim H, Bohr Y, Connolly J. Prevalence and characteristics of teen motherhood in Canada. Maternal Child Health. 2012;16(1):228-234.
15. Sinha M. La violence familiale au Canada : un profil statistique 2011. Juristat. Ottawa (Ont.) : Statistique Canada, Centre canadien de la statistique juridique; 2013. [Statistique Canada, $\mathrm{N}^{\mathrm{o}} 85-002$ au catalogue].

16. Cederbaum JA, Putnam-Hornstein E, King B, Gilbert K, Needell B. Infant birth weight and maltreatment of adolescent mothers. Am J Prev Med. 2013;45(2):197-201.

17. Noll JG, Shenk CE. Teen birth rates in sexually abused and neglected females. Pediatrics. 2013;131(4):e1181-e1187.

18. Valentino K, Nuttall AK, Comas M, Borkowski JG, Akai CE. Intergenerational continuity of child abuse among adolescent mothers: authoritarian parenting, community violence, and race. Child Maltreat. 2012;17(2):172-181.

19. Thornberry TP, Matsuda M, Greenman SJ et collab. Adolescent risk factors for child maltreatment. Child Abuse Negl. 2014;38(4) 706-722.

20. Olds DL, Kitzman H, Knudtson MD, Anson E, Smith JA, Cole R. Effect of home visiting by nurses on maternal and child mortality: results of a 2-decade follow-up of a randomized clinical trial. JAMA Pediatr. 2014; 168(9):800-806

21. Olds DL. The Nurse-Family Partnership: an evidence-based preventive intervention. Infant Ment Health J. 2006;27:5-25.

22. Zielinski DS, Eckenrode J, Olds DL. Nurse home visitation and the prevention of child maltreatment: Impact on the timing of official reports. Dev Psychopathol. 2009;21:441-453.

23. Olds DL, Sadler S, Kitzman H. Programs for parents of infants and toddlers: recent evidence from randomized trials. J Child Psychol Psychiatry. 2007;48:355-391.

24. Jack SM, Catherine N, Gonzalez A et collab. Adaptation, mise à l'épreuve et évaluation d'interventions complexes en santé publique: leçons tirées du Nurse-Family Partnership dans le secteur de la santé publique au Canada. Promotion de la santé et prévention des maladies chroniques au Canada. 2015;35(8/9):161-70. 